\title{
Clear Cell Papulosis: A Case Report
}

\author{
So-Woon Kim · Jin Roh · Chan-Sik Park \\ Department of Pathology, Asan Medical Center, University of Ulsan College of Medicine, Seoul, Korea
}

Clear cell papulosis (CCP) is an extremely rare entity, as only about two dozen cases have been reported to date in the English literature. ${ }^{1}$ In CCP, white papules arise and cluster bilaterally along the mammary line, usually over the lower abdomen and pubis. ${ }^{2}$ There is a predisposition for persons of Asian or Hispanic descents and most cases have been found in healthy children younger than 6 years old. ${ }^{1,3}$ Typically, the lesions spontaneously resolve with age. ${ }^{4}$ Microscopically, the lesions are composed of aberrant cells derived from sweat gland epithelial cells that grow in a pagetoid pattern in the epidermis. ${ }^{2}$ CCP provides evidence for the potential precursor cells for cutaneous Paget's disease. ${ }^{5}$ Pathologists should include CCP in the differential diagnosis of whitish maculopapular lesions in the mammary line.

\section{CASE REPORT}

A 2-year-old Korean girl presented with multiple scattered white papules on her lower abdomen and pubic areas, which had gradually increased in size. The lesions were asymptomatic with no pruritus, pain, or sensory impairment. She exhibited no other remarkable medical conditions. A physical examination revealed many sharply demarcated, round-to-oval hypopigmented maculopapules that were predominantly located in the suprapubic area (Fig. 1).

A punch biopsy was obtained from one of the lesions. Microscopically, the lesion showed mild acanthosis and reduced pigmentation of the epidermis (Fig. 2A). A characteristic feature was the presence of benign pagetoid clear cells in the epidermis.

\section{Corresponding Author}

Chan-Sik Park, MD

Department of Pathology, Asan Medical Center, University of Ulsan College of

Medicine, 88 Olympic-ro 43-gil, Songpa-gu, Seoul 05505, Korea

Tel: +82-2-3010-5838, Fax: +82-2-3010-8675, E-mail: csikpark@amc.seoul.kr

Received: December 28, 2015 Revised: February 2, 2016

Accepted: February 16, 2016
The clear cells occurred either in clusters or singly along the basal and suprabasal layers of the epidermis and were less frequent near the surface. There was no involvement of the dermis. The aberrant cells were larger than keratinocytes and had abundant pale eosinophilic to clear cytoplasm without pigmentation (Fig. 2B). Nuclei were round-to-oval without pleomorphism. Nucleoli were not prominent. No mitosis was observed. The abnormal cells were positive for cytokeratin 7 and carcinoembryonic antigen (Fig. 3A, B) by immunohistochemical analyses. By contrast, Melan A and S100 protein stains were negative. Only epidermal melanocytes and dendritic cells showed positive staining for Melan A and S100 protein, respectively (Fig. 3C, D). Epidermal keratinocytes were negative for all stains.

\section{DISCUSSION}

We here report a case of CCP, which is an extraordinarily rare condition. CCP is almost always restricted to the skin without systemic symptoms. ${ }^{4}$ Lesions can be easily missed without suspicion because the histologic findings are not readily discernible to pathologists who are not familiar with this entity. ${ }^{1}$ In such cases, the diagnosis can be confirmed pathologically by immunostaining. ${ }^{6}$

The exact reason for the hypopigmented change remains unknown but may be a consequence of reduced melanization in the epidermis. Additionally, the number of melanocytes is normal or slightly reduced. These findings suggest the dysfunction of melanocytes in lesions, although the role of clear cells in the pathogenesis of this type of melanocytic dysfunction remains to be elucidated. ${ }^{6}$

The differential clinical diagnoses of a hypopigmented lesion include vitiligo, chicken pox scars, idiopathic guttate hypomelanosis, hypomelanotic tinea versicolor, and anetoderma. ${ }^{7}$ In a typical clinical setting and with microscopic findings that show no 

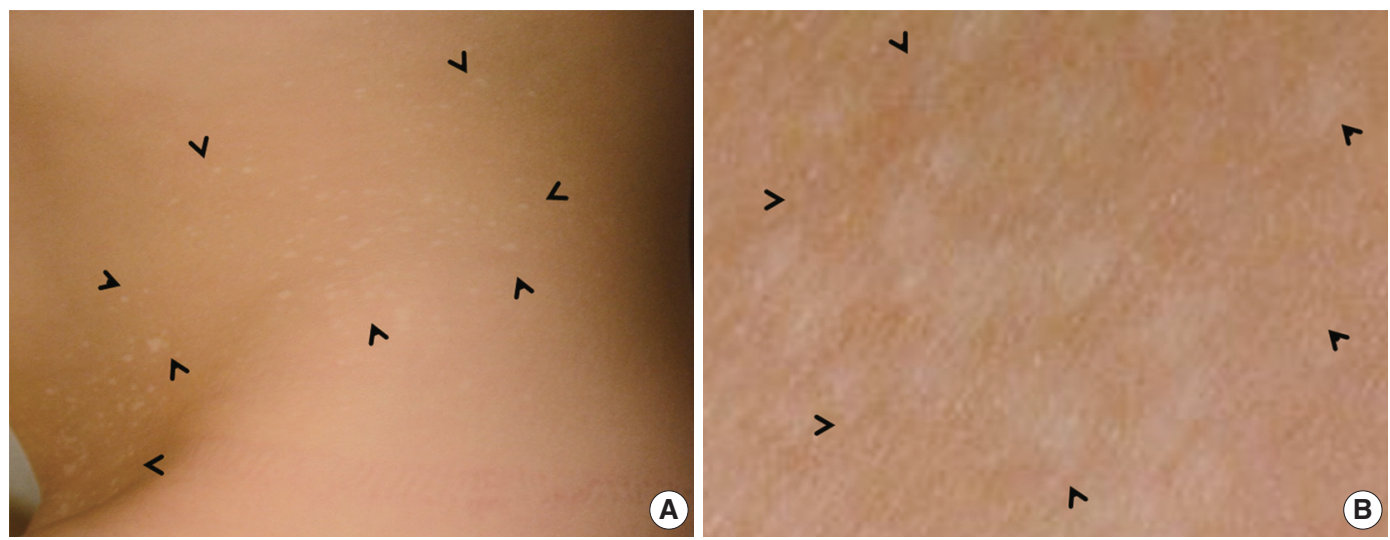

Fig. 1. (A) Many small whitish macules are present on the trunk and are particularly numerous in the lower abdomen and pubic area (arrowheads). The milk-line distribution of the lesions is notable. (B) Close-up view of the right pubic areas showing round-to-oval hypopigmented with no scales (arrowheads).
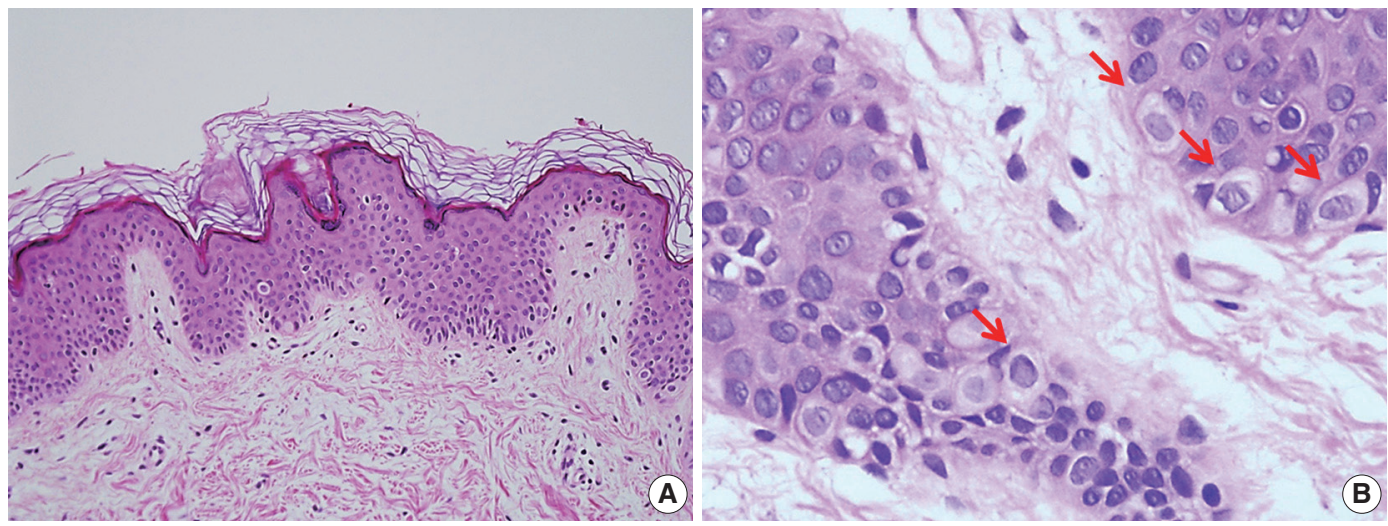

Fig. 2. Biopsy of a clear cell papulosis lesion revealing scattered single clear cells in the basal layer with mild acanthosis and no alteration of the keratin layer. (A) There is minimal inflammatory cell infiltration in the dermis. (B) At higher magnification, solitary benign-appearing pagetoid cells with an abundant clear cytoplasm could be observed in the epidermis. No nuclear atypia is noted (arrows, clear cell).

clear cells in basal layer, CCP can usually be differentiated from the aforementioned diseases. Histological differential diagnoses include the early stages of extramammary Paget disease, pagetoid squamous carcinoma, pagetoid melanoma, and sebaceous carcinoma. ${ }^{7}$ All of these conditions have malignant cytologic features, whereas the clear cells of CCP clearly appear to be benign. ${ }^{3}$ The histological and immunohistochemical staining patterns suggest that the affected cells in CCP are most likely to be of sweat gland epithelial cell origin that are derived from Toker cells. ${ }^{8,9}$ However, the natural history of CCP remains unknown. Notably, there is another Toker cell proliferative lesion called Toker cell hyperplasia (TCH), which is usually found nearby extramammary Paget disease. This topographical relation suggested that TCH may be a precursor lesion for extramammary Paget disease. ${ }^{5}$ There is no discernible histomorphologic difference between TCH and CCP besides clinical features. Usually, TCH is clinically unremarkable. Her2/neu immunostaining shows faint positivity in some TCH but negativity in CCP. Mucin stains are consistently negative in TCH but variably positive in CCP

In conclusion, the recognition of hypopigmented lesions in the mammary line is important in clinical practice. CCP should be included in differential diagnoses of both hypopigmented lesions and depigmented extramammary Paget disease. To the best of our knowledge, our current case study represents the first report of CCP in the Korean literature. Awareness and making an accurate diagnosis of CCP may mitigate overly aggressive management.

\section{Conflicts of Interest}

No potential conflict of interest relevant to this article was reported. 

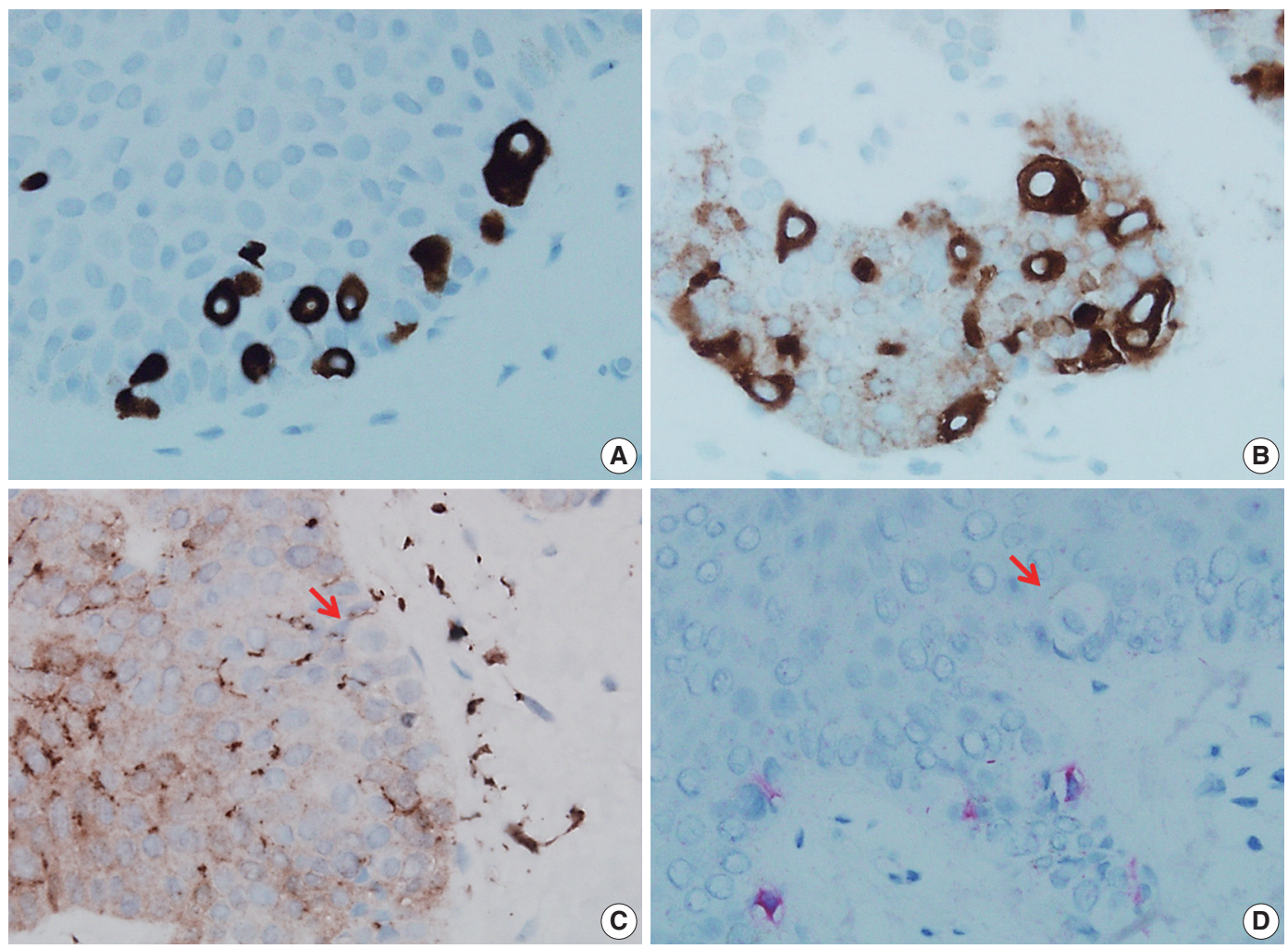

Fig. 3. The pagetoid cells strongly express cytokeratin 7 (A) and carcinoembryonic antigen (B) but are negative for either S100 protein (C) or Melan A (D) (arrows, clear cell).

\section{REFERENCES}

1. Silverberg NB. Clear cell papulosis. In: Silver NB, Duran-McKinster C, Tay YK, eds. Pediatric skin of color. New York: Springer, 2015; 229-30.

2. Kuo TT, Chan HL, Hsueh S. Clear cell papulosis of the skin. A new entity with histogenetic implications for cutaneous Paget's disease. Am J Surg Pathol 1987; 11: 827-34.

3. Mohanty SK, Arora R, Kakkar N, Kumar B. Clear cell papulosis of the skin. Ann Diagn Pathol 2002; 6: 385-8.

4. Tseng FW, Kuo TT, Lu PH, Chan HL, Chan MJ, Hui RC. Long-term follow-up study of clear cell papulosis. J Am Acad Dermatol 2010; 63: 266-73.

5. Yu Y, Sukhatme S, Loo DS. Clear cell papulosis: a connection of clear cells to toker cells or paget disease. Arch Dermatol 2009; 145: $1066-8$.

6. Wysong A, Sundram U, Benjamin L. Clear-cell papulosis: a rare entity that may be misconstrued pathologically as normal skin. Pediatr Dermatol 2012; 29: 195-8.

7. Chen YH, Wong TW, Lee JY. Depigmented genital extramammary Paget's disease: a possible histogenetic link to Toker's clear cells and clear cell papulosis. J Cutan Pathol 2001; 28: 105-8.

8. Kim YC, Mehregan DA, Bang D. Clear cell papulosis: an immunohistochemical study to determine histogenesis. J Cutan Pathol 2002; 29: 11-4.

9. Di Tommaso L, Franchi G, Destro A, et al. Toker cells of the breast: morphological and immunohistochemical characterization of 40 cases. Hum Pathol 2008; 39: 1295-300. 